TENDENCIAS

Revista de la Facultad de Ciencias

Económicas y Administrativas.

Universidad de Nariño

ISSN-E 2539-0554

Vol. XXII No. 2 - 2do Semestre 2021

Julio-Diciembre - Páginas 309-330

\title{
EL CAPITAL INTELECTUAL EN LAS ORGANIZACIONES DE ECONOMÍA SOLIDARIA
}

\section{INTELLECTUAL CAPITAL IN SOLIDARITY ECONOMY ORGANIZATIONS}

\section{CAPITAL INTELECTUAL EM ORGANIZAÇÕES DE ECONOMIA SOLIDÁRIA}

Carlos Augusto Rincón Soto; Francisco Ricardo Molina Mora; Patricia González González

Magíster en Contabilidad, Universidad del Valle, Colombia; Magíster en Proyectos Universidad Internacional Iberoamericana, Puerto Rico. Docente del Programa de Contaduría Pública, Universidad del Valle. ORCiD: 0000-0003-4710-5558. E-mail: carlos.augusto.rincon@correounivalle.edu.co, Colombia.

Magíster en Administración Universidad del Valle, Colombia. Docente del Programa de Contaduría Pública, Universidad del Valle. ORCiD: 0000-0003-1998-3492. E-mail: francisco.molina@correounivalle.edu.co, Colombia.

Doctora en Contabilidad y Contraloría, Universidad de Sao Pablo. Docente del Programa de Contaduría Pública, Universidad del Valle. ORCiD: 0000-0002-7396-3401. E-mail: patricia.gonzalez@correounivalle.edu.co, Colombia.

Recibido: 18 de noviembre de 2020

Aprobado: 12 de mayo de 2021

DOI: https://doi.org/10.22267/rtend.212202.178

\section{Resumen}

El objetivo de este artículo es aportar a la discusión sobre como el capital solidario hace parte de la estructura del capital intelectual en las empresas de economía solidaria. Esto es importante, dado que al develar este intangible, incentiva su control y gestión, así como su respectiva investigación, 
lo que crea estrategias para optimizarlo, de modo que, esto podría clarificar los fines y mejorar los resultados de las entidades de economía solidaria. Este artículo desarrolla un marco conceptual y una revisión de literatura, así como un análisis interpretativo de los resultados. Los hallazgos mostraron que la literatura reconoce la existencia de otro factor en la economía solidaria, además, se encontró que la literatura sobre el factor C podría aportar a la descripción y elaboración de la caracterización de capital solidario.

Palabras clave: activo inmaterial; colectivismo; cooperativa; economía colectiva; factor C.

JEL: B50; B55; E22; O49; P13

\begin{abstract}
The objective of this article is to contribute to the discussion on how the capital of solidarity is part of the structure of intellectual capital in companies with solidarity-based economy. This is important, since by unveiling this intangible, it encourages its control and management, as well as its correspondent research, which creates strategies to optimize it, so that this could clarify the purposes and improve the results of the entities with solidarity-based economy. This article elaborates a conceptual framework and a literature review, as well as an interpretive analysis of the results. The findings showed that the literature recognizes the existence of other factor in the solidarity-based economy, in addition, it was found that the literature on factor $\mathrm{C}$ could contribute to the description and elaboration of the characterization of the capital of solidarity.
\end{abstract}

Keywords: intangible asset; collectivism; cooperative; collective economy; factor C.

JEL: B50; B55; E22; O49; P13

\title{
Resumo
}

O objetivo deste artigo é contribuir para a discussão sobre como o capital solidário é parte na estrutura do capital intelectual nas empresas de economia solidária. Isso é importante, pois ao desvendar esse intangível, incentiva seu controle e gestão, assim como suas respectivas pesquisas, que criam estratégias para otimizá-lo, de forma que possa se esclarecer os propósitos e melhorar os resultados das entidades de economia solidária. Este artigo desenvolve uma estrutura conceitual e uma revisão da literatura, assim como uma análise interpretativa dos resultados. As evidências mostram que a literatura reconhece a existência de outro fator na economia solidária, além disso, 
constatou-se que a literatura sobre o fator $\mathrm{C}$ pode contribuir para a descrição e elaboração da caracterização do capital solidário.

Palavras-chave: ativo intangível; coletivismo; cooperativa; economia coletiva; fator C.

JEL: B50; B55; E22; O49; P13

\section{Introducción}

En el modelo utilitarista el beneficio de las empresas se mide a partir de las ganancias (Goldratt, 1994; Goldratt et al., 2008). En cambio, en las empresas de economía solidaria, el fin de la organización se basa en una jerarquía, donde es más importante el bienestar común de los asociados. Los factores económicos que añaden valor a la producción de tales fines, no se representan plenamente en los estados financieros. Los activos revelan una caracterización de los bienes de la empresa en tangibles, financieros e intangibles adquiridos, que son aportados por terceros, o por los dueños (Delgadillo, 2001). Por lo general, el valor de mercado de las empresas es más alto que el valor contable, permitiendo reconocer que existen otros factores extracontables que no están relacionados en los estados financieros (Chen et al., 2005) y que comúnmente, son bienes inmateriales difíciles de medir y valorar (Funes y Hernández, 2001; Reilly y Schweihs, 1998). En economía se les reconoce como bienes intangibles formados, es decir, el capital intelectual (CI) de las empresas. Estos bienes intangibles teorizados como conocimientos y relaciones sociales, añaden, en algunas situaciones, mayor valor que los bienes tangibles, por esto ha sido indispensable que en los programas de investigación sobre CI se describan, interpreten, comprendan y se sometan a medición.

Al describir el activo intangible como CI (Keong, 2008), se ha generado una variedad de categorías que buscan formular un concepto, para distinguirlo e incluirlo en los objetivos de la empresa. La categorización más aceptada sobre CI, distingue tres factores: capital humano, estructural y relacional (Ragab y Arisha, 2013; Galleguillos-Cortés et al., 2018; Iazzolino y Laise, 2016; Mačerinskienė y Survilaitè, 2019; Ståhle et al., 2011).

Algunos autores agregan el capital social como un factor importante que representa elementos extracontables y que añaden valor a la organización (Ostrom y Ahn, 2003; Tamošiūnienè et al., 
2015), potenciadores de valor como la confianza, lealtad, sinceridad, transparencia, compromiso, solidaridad, responsabilidad, honestidad y ética a las relaciones sociales de la organización (Bueno, 2002).

El concepto de capital social es muy amplio y caracterizarlo puede ser complejo debido a las significaciones en torno a "lo social", que por lo general se definen en relación con la sociedad (Campos, 2008). Millán y Gordon (2004) coinciden en que el capital social consiste en recursos que derivan de la sociedad misma. Ostrom y Ahn (2003) por su parte plantean que este "Concepto es ambiguo porque es demasiado ambicioso" (p. 156). Otros autores establecen lo social dentro del capital humano y relacional (Tamošiūnienè et al., 2015) y en algunas caracterizaciones contables a los aportes de los accionistas se llama capital social (Aparicio, 2003; Walde, 2011; Presidencia de la República de Colombia, 2005), ampliando el concepto a bienes tangibles, financieros e intangibles.

La caracterización común de CI se recoge en la caracterización de los factores de capital humano, estructural y relacional, pero el capital social parece no estar incorporado en ninguno de ellos. En las empresas de economía solidaria existe un intangible extracontable, diferente de la caracterización común del CI, que añade valor a estas organizaciones. Por lo tanto, este trabajo pretende concertar y adherir un tipo de factor relacionado con el capital social, el cual se puede observar con claridad en la economía solidaria y es, habitualmente, superficial para las empresas de economía utilitarista. Entonces, el objetivo de este artículo es aportar a la discusión sobre como el capital solidario hace parte de la estructura del capital intelectual en las empresas de economía solidaria.

Esto es importante dado que conocer los factores que añaden valor a las distintas formas organizacionales, permite crear estrategias para gestionarlos, determinando mayor rendimiento; por el contrario, ignorar la existencia de los mismos en la organización, puede llevar a orientaciones y decisiones erróneas sobre los atributos de estos factores e impactarlos negativamente, disminuyendo el valor de la empresa. 
Este artículo de reflexión está divido en cinco secciones, primero, introducción; segundo la metodología, en la tercera sección se expone la estructura del CI; cuarto se describe el concepto de capital solidario; quinto, aborda el concepto de capital solidario dentro de la estructura del CI para las empresas de economía solidaria, y por último, se presentan discusiones y conclusiones.

\section{Metodología}

Para Vera (2009) el artículo de revisión es considerado un estudio pormenorizado, selectivo y crítico, que recopila la información más relevante de un tema específico. Por consiguiente, se realizó una revisión de la literatura de documentos que tuvieran como criterio el análisis de capital social, capital solidario, los principios cooperativos y la solidaridad como un elemento intangible (Arriagada, 2006; Marcuello y Saz, 2008). Para ello, se realizó la búsqueda en distintas bases de datos como: Scopus, ScienceDirect, y otras de acceso libre como Scielo, Redalyc, Latindex, Dialnet, SSRN. Para este ejercicio se excluyeron aquellos textos que trabajan el tema de contabilidad social desde una mirada exclusivamente contable y financiara.

Además, se realizó un análisis interpretativo de los documentos, la cual busca conceptualizar y darle sentido a los fenómenos estudiados (Packer, 2013). Por ello, se utilizó esta metodología con la intención de comprender el papel que cumple el capital solidario en las organizaciones de economía solidaria, y como este, podría ser un factor importante en el capital intelectual de las entidades de economía solidaria.

\section{Resultados}

\section{El capital intelectual}

Una empresa está compuesta por inversiones en activos tangibles, intangibles adquiridos y financieros. La representación isomorfa contable es igual a: Inversión = Financiación (Delgadillo, 2001; González, 2017); en la ecuación contable se representa como: Activo = Pasivo + Patrimonio (Ijiri, 1967; Mattessich, 2002). Las inversiones representan los activos, que se financian por medio de pasivos y patrimonio; dentro del patrimonio se encuentra el capital, que se establece como los aportes de los socios, por lo tanto, hay una parte de los activos, financiada por 
el capital; dentro del patrimonio hay otras partidas distintas al capital, que no son aportes, pero hacen parte de la propiedad de los dueños; algunas de esas partidas se pueden repartir o capitalizar; las partidas del patrimonio mientras estén en la organización, son potencialmente capital, por lo tanto, el capital identifica los valores de los derechos de propiedad de los dueños, que se utiliza para crear valor dentro de los objetivos de la organización.

Para Rodov y Leliaert (2002) los estados financieros solo revelan los costos históricos de los activos comunes, pero dejan por fuera los intangibles formados (un activo relevante para la producción de la rentabilidad de las organizaciones) quedando oculto en la información financiera el valor que las personas aportan a la organización.

Para algunos autores, la diferencia está sustentada en el valor de los intangibles que la contabilidad no revela (Keong, 2008; Reilly y Schweihs, 1998). El problema actual, es que la información contable con la que se toman decisiones financieras de las empresas no justifica esta diferencia, porque no estima el potencial de los activos intangibles. La normatividad contable solo reconoce los intangibles adquiridos y deja como gasto los intangibles formados (IFRS Foundation, 2019). El conocimiento desarrollado por la organización es clave para la revelación del valor futuro agregado de la empresa, por lo tanto, el valor futuro depende de los intangibles (Rodov y Leliaert, 2002). El valor en libros se sustenta en los costos históricos, en cambio el valor de mercado reconoce la capacidad productiva futura. ${ }^{1}$ Esto determina la diferencia entre valor en libros contables y el valor de mercado. La contabilidad deja un vacío en la explicación de la diferencia, que es asumida por los investigadores del CI.

Los activos intangibles se han llamado activos invisibles, recurso intangible, propiedad intelectual, CI, valor inmaterial, y se han definido como activos no físicos, no financieros, no monetarios, no corrientes, pues poseen valor o le añaden valor a otros activos tangibles; consisten en conocimientos que producen ingresos, es decir, se trata de un valor invisible, no tiene existencia física, pero cuenta con potencial para producir ingresos o disminuir costos (Keong, 2008). Los

\footnotetext{
${ }^{1}$ Si bajo NIIF las empresas contabilizan al VR, los modelos que valoran el intangible basados en la diferencia entre el valor en libros y valor de mercado, no tendrían justificación, porque parte del valor intangible quedaría intrínseco en el VR. Este artículo no aborda este problema.
} 
activos tangibles por sus características de poseer cuerpo físico, ser contables y medibles, son mucho más fáciles de ser identificados, en cambio los intangibles, por ser inmateriales, son más difíciles de identificar.

Los investigadores han definido el CI como la diferencia entre el valor de mercado de la empresa y el valor en libros de la contabilidad, impulsado por goodwill, el cual puede ser adquirido o formado. En las NIIF, el goodwill adquirido es el único aceptable para la información contable. En cambio, los consumos realizados en un goodwill formado, se contabilizará como un gasto y se revelará en el estado de resultados.

El goodwill comprado, se podrá activar en la contabilidad y amortizarse durante la vida útil del activo. Sin embargo, el activo formado, no será reconocido para la contabilidad, a menos que sea un activo de investigación y desarrollo que esté en fase productiva (IFRS Foundation, 2019). Esto deja por fuera de la contabilidad, una cantidad de activos intangibles y factores importantes que le añaden valor a la empresa. Por esa razón, hay una diferencia entre el valor de mercado de la empresa y el valor contable: el primero reconoce el valor de los activos contables más los activos intangibles.

En la economía basada en el conocimiento, el CI se ha convertido en la clave para analizar la creación de valor. Keong (2008) en acuerdo con Andreou et al. (2007) declaran que en la era del conocimiento, la inteligencia se convierte en un recurso importante para la producción de ingresos. Para Reilly y Schweihs (1998) los análisis de activos intangibles pueden ser útiles como una herramienta para entender la capacidad proyectada de ganancias, la facultad generadora de flujo de efectivo y la vida funcional, tecnológica o económica asociada con la propiedad intangible.

Los estudios de CI son desarrollados con la intención de conocer el impacto de los activos intangibles en los negocios, pues se ha aceptado que los activos intangibles aportan al crecimiento de la rentabilidad de la empresa, pero su medición no es tan sencilla porque no hay una relación directa y unilateral, entre gasto en activos intangibles e ingresos (Ragab y Arisha, 2013). 
Para Pulic (2008) desarrollador del coeficiente de valor añadido (VAIC), el CI suma a la creación del valor de las empresas, por lo que la eficiencia se determina en la creación de ganancias. En la economía actual, la actividad predominante ya no es la producción de bienes sino la producción de conocimiento, que se integra en bienes y servicios.

De la misma manera que se hicieron inversiones en plantas y maquinarias para crear valor durante la economía industrial, hoy invertimos en empleados, que son los principales creadores de valor de la economía contemporánea. Hoy en día, las empresas combinan dos recursos clave: capital físico y financiero e intelectual. (Pulic, 2008, p. 5)

Este autor manifiesta que el conocimiento sirve como elemento clave en la toma de decisiones. El conocimiento como "capacidad de actuar" está destinado a un sentido práctico e intelectual. El intercambio del contrato de mano de obra o costo de personal, es entonces el costo de contratación del potencial de acciones dado por los conocimientos de las personas, que se derivan en los resultados financieros que espera obtener la empresa, pues la eficiencia esperada en las utilidades con respecto al capital humano, debe ser capaz de producir rendimientos para pagar por lo menos el costo de la contratación del recurso humano y su potencial de decisiones y actuaciones en favor de la producción de utilidades.

En los últimos años, muchos individuos y grupos de diferentes disciplinas han intentado acordar una definición estándar para CI. Algunos autores plantean que el conocimiento se puede convertir en valor, siendo una experiencia que se usa para crear riqueza desarrollando una ventaja competitiva en las organizaciones (Nazari y Herremans, 2007). Cuando los activos intangibles y tangibles se complementan y se utilizan eficazmente, pueden crear riqueza al producir un activo de mayor valor, llamado CI.

Comenzando la década de 1960, Gary Becker, ganador del Premio Nobel de Ciencias Económicas de 1992, reconoció la importancia de la experiencia humana, argumentando que "Los gastos en educación, capacitación, y atención médica, [...] producen capital humano" (Nazari y Herremans, 2007, p. 3). 
Para Iazzolino y Laise (2016) la productividad de los trabajadores del conocimiento es igual a la relación entre el valor agregado (VA) por el trabajador y su salario, pues son las decisiones que se toman, en función del conocimiento, lo que permite obtener los resultados financieros. Entonces, los resultados financieros, son eficiencias de las decisiones y, por lo tanto, del conocimiento intelectual.

Un mayor conocimiento intelectual se relaciona con mejores decisiones para el crecimiento económico, de modo que los salarios de las personas son inversiones y no meros egresos, porque de estas inversiones se espera rentabilidad. La diferencia entre la inversión y la rentabilidad, es la eficiencia de la inversión, que es lo que mide el coeficiente de eficiencia VAIC (Iazzolino y Laise, 2013). Entre mayor inversión en CI, mayor debe ser la rentabilidad, aumentando la eficiencia. Estos autores afirman que, para incrementar la productividad del conocimiento, se debe invertir en trabajadores con capacidades intelectuales que agreguen valor en mayor medida de lo que cuestan. "Para descubrir la eficiencia del CI, es necesario identificar los procesos que destruyen valor, que son los que funcionan por debajo del promedio de eficiencia de la compañía" (Pulic, 2008, p. 14).

La reducción de la inversión en CI, fácilmente se vería revelada como una disminución en la capacidad productiva de las empresas tecnológicas. Hoy, sin la inversión en nuevo conocimiento, las empresas no podrían subsistir a largo plazo. Invertir en el personal es una estrategia "ganarganar", porque motiva e incentiva a los empleados a crear mejores propuestas y decisiones, que se verán recompensadas en rentabilidad. Por el contrario, cuando solo se piensa en aumentar la eficiencia de los accionistas, disminuyendo la inversión en los trabajadores (rentabilidad de los trabajadores), existe desmotivación y sus posibilidades y capacidades disminuirán su eficiencia (Iazzolino y Laise, 2016).

Chen et al. (2005) expone que las limitaciones en los estados financieros para explicar el valor de las empresas, es el hecho que la fuente del valor económico ya no es la producción de bienes materiales, sino la creación de CI. 


\section{Estructura del capital intelectual}

Clasificar la información sobre el capital intelectual es una labor compleja, la literatura de caracterización de los activos intangibles, proporciona una orientación que ha permitido elaborar una taxonomía de los activos intangibles, al igual que desarrollar un soporte teórico, que brinda consistencia y coherencia a esa clasificación, y ha servido para homogenizar los reportes de los activos intangibles.

La clasificación supone un más alto grado de complejidad, pues la podemos definir como la ordenación hecha aplicando un método. En este método va implícito un proceso de comparación de los elementos a clasificar, para determinar sus similitudes y diferencias, que son las que van a determinar la posición de unos elementos con respecto a otros. (Currás, 2005, p. 53)

Para Reilly y Schweihs (1998) existe una forma muy global de catalogar los activos intangibles con: goodwill y know how. Se ha planteado que una mayor discriminación de estas clases de activos podría facilitar una mejor comprensión de ellos, pues cada uno tiene elementos particulares que los hace especiales, lo cual podría influir en la forma de estimarlos, porque agruparlos en categorías tan globales, no permite observar atributos y características importantes.

Según Reilly y Schweihs (1998) los activos intangibles, se categorizan comúnmente en los siguientes:

a. Relacionados con el mercado (marcas, nombres comerciales, registros, logos)

b. Relacionados con la tecnología (patentes, documentación técnica, notas de laboratorio, knowhow)

c. Relacionadas con el arte (trabajos literarios, derechos de autor, composición musical, mapas, etc.)

d. Relacionados con procesamiento de datos (software, database, etc.)

e. Relacionados con la ingeniería (diseños, patentes de productos, secretos de mercado, etc.)

f. Relacionados con contratos (licencias, franquicias, acuerdos, etc.)

g. Relacionados con el recurso humano (acuerdos con empleados, montajes de personal, etc.) 
h. Relacionados con la ubicación (derechos de explotación minera, derechos de volar en un área, derechos sobre el agua, etc.)

i. Relacionados con el buen nombre (celebridades, persona, goodwill, etc.).

Un activo intangible se puede relacionar con una o varias de las categorías presentadas; es importante comprender su caracterización, pues de ello también depende su valorización.

Keong (2008) propone un barrido de las propuestas de clasificación del capital intelectual y expone que Hall (1992) divide los activos intangibles en dos categorías: propiedad intelectual y activos de conocimiento; Brookin (1997) los identifica como 1. Capital humano, 2. Capital organizacional, 3. Capital del cliente; para Stewart (1998): 1. Capital humano, 2. Capital estructural, 3. Capital del cliente; Lev (2001) propone igualmente tres categorías: 1. Innovación, 2. Recursos humanos, 3. Prácticas organizacionales; Gu y Lev (2001) proponen cinco categorías: 1. Investigación y desarrollo, 2. Publicidad, 3. Gastos de capital, 4. Sistemas de información y, 5. Adquisición de tecnología; Bukh et al. (2001) compara varias de estas taxonomías y concluye en tres categorías: 1. Actividades conectadas con los empleados, 2. Tareas, procedimientos y estructuras y 3. Servicios y valor añadido a actividades relacionadas con los clientes.

Keong (2008) considera que se pueden relacionar otras clasificaciones como es la categorización en cuatro principales clases: activos de mercado, activos relacionados con el capital humano, activos de propiedad intelectual y activos de infraestructura. La propuesta por $\mathrm{KPMG},{ }^{2}$ basada en la metodología contable, caracteriza los intangibles en cinco tipos: activos y dotaciones, habilidades y conocimiento tácito, valores colectivos y normas, tecnología y conocimiento explícito y procesos primarios y de gestión (Keong, 2008).

El coeficiente de valor añadido VAIC propuesto por Pulic (2008), sugiere una metodología de medición de la eficiencia del capital humano y estructural; el Skandia Navigater propone dos clases: capital humano y estructural, subdividiendo el capital estructural en capital organizacional y clientes (Edvinsson, 1997); la metodología FIMIAN propone una valoración desde la

\footnotetext{
${ }^{2}$ KPMG: Red global de firmas de servicios profesionales en auditoría, asesoramiento financiero y contable.
} 
ponderación de pesos de importancia de los intangibles, divididos en capital humano, relacional y estructural, aplicado a la diferencia del valor de mercado y el contable (Rodov y Leliaert, 2002); el VAIC extendido propone la clasificación en capital humano, relacional y estructural, y una subdivisión para el estructural, en procesos, investigación y desarrollo (Nazari y Herremans, 2007). Survilaite et al. (2015) propone una división del CI en estructura externa, estructura interna y capital social. Así, la clasificación de los elementos que componen el CI, por características y atributos, antecede a la valoración, pues no se puede valorar, ni medir lo que no se conoce, ordena y organiza. Aunque existe un trabajo interesante en la primera fase de clasificación de los factores del CI, esta teoría, aun es temprana y todavía debe permitir integrar nuevas propuestas y enfoques (Andriessen, 2001; Survilaitė et al., 2015).

Para algunos autores, existen tres categorías en las que se ha concentrado la definición de capital intelectual: 1. Capital humano, 2. Estructural, 3. Capital relacional (Ragab y Arisha, 2013; Galleguillos-Cortés et al., 2018; Mačerinskienė y Survilaite, 2019; Nazari y Herremans, 2007). Cada uno de estos componentes está formado por varios factores, por ejemplo, la estructura del capital humano está compuesta por factores como reputación, innovación, competencia, experiencia, habilidades, antigüedad, etc.

Según Rodov y Leliaert (2002) los factores se pueden relacionar con varios de los componentes. El capital humano es el principal factor para desarrollar capital estructural, este es relativo a los empleados, y el capital estructural a las organizaciones. Aunque el capital estructural es creado por el capital humano, existe de manera independiente; por ejemplo: las patentes, marcas, procesos, etc. Son creadas por capital humano, pero después de la creación y demarcación de derechos de propiedad, pertenecen a la compañía.

El capital relacional se define como la capacidad de una organización para interactuar positivamente con los miembros de la comunidad empresarial: clientes, proveedores, prestamistas, organismos gubernamentales, etc. Básicamente, el capital relacional depende de las dinámicas y la efectividad de la relación con los clientes. El capital humano y el capital estructural deben ayudar a crear capital relacional. 
La medición del aporte del capital intelectual al desempeño de la organización es importante para la toma de decisiones, asignación de recursos y desarrollo de proyectos. Sin embargo, la medición del capital intelectual todavía se encuentra en una etapa exploratoria. Los estudios sugieren que las medidas de desempeño no financiero son mejores predictores del rendimiento a largo plazo y, por lo tanto, deberían ser utilizados para ayudar a los administradores a enfocarse en los aspectos proyectivos de sus decisiones (Pedro et al., 2018; Survilaitè et al., 2015).

La elaboración de la estructura taxonómica del capital intelectual es un aporte de la comunidad de investigadores, quienes contrastan ideas para lograr acordar una clasificación que se adecúe con mayor precisión a la realidad del objeto de estudio. La teorización de la clasificación debe cumplir con la teoría de conjuntos, donde el dominio de los elementos debería estar claramente definido.

\section{El factor C como un componente del capital intelectual: capital solidario}

La teoría económica clásica reconoce tres factores de producción: la tierra, el trabajo y el capital (Smith, 1776). La teoría neoclásica suprimió la tierra, por considerarla parte del capital (Agudelo et al., 2010). La economía basada en el conocimiento reconoce el trabajo como un factor clave en la producción de CI (Castells, 2002). Este se plantea como capital humano, que integra el conocimiento que ayuda a crear capital relacional y capital estructural.

Para Razeto (1996) en las empresas de economía solidaria, existe otro factor que no aparece en las empresas del sector real. Al estudiar empresas comunitarias, analiza que los factores de capital financiero, tangible y conocimiento intelectual eran muy limitados para explicar los elementos que aportan valor, además, los trabajadores no gozaban de capacitación académica y sus habilidades eran consideradas ineficientes por el mercado, pero cuando evaluaban los resultados, con respecto a los factores económicos tangibles, eran altamente productivas y rentables.

Los medios materiales con que trabajaban y el financiamiento, o sea, el capital es mínimo, casi producen sin capital [...] la tecnología, el saber técnico de las señoras, de los jóvenes, de las personas que trabajan en esas organizaciones era un saber parcial, muy insuficiente, incompleto $[\ldots]$ la fuerza de trabajo no era la más productiva porque las experiencias las 
formaban personas que no encontraban trabajo, porque en realidad sus fuerzas productivas eran menos eficientes [...] el financiamiento ni hablar, porque era insignificante [...] la gestión: no eran personas acostumbradas a tomar decisiones, que hubieran hecho estudios de administración de empresas, que fueran ejecutivos, que tuvieran capacidad. (Razeto Migliaro, 2007a, p. 5)

Los resultados se deben a otro factor, que no es el capital financiero tangible, ni de conocimiento (Escobar et al., 2018). Enfatiza en reglas morales de conducta, que hacen que las personas se valoren como un elemento importante de la comunidad. Razeto (2015) lo llamó factor C, porque muchas de las palabras relacionadas para identificarlos, comenzaban con la letra $\mathrm{C}$ : cooperación, comunicación, cordialidad, colectividad, carisma, etc. (Agudelo et al., 2010). Además de democracia, amor, pertenencia, propiedad, felicidad, emprendimiento, solidaridad, aptitudes y actitudes que se acoplan para crear una empresa cooperativa.

El "factor C" podría relacionarse con el capital social y solidario (Ruiz y Quesada, 2014; Marcuello y Saz, 2008), el cual se desarrolla bajo un clima organizacional de cooperación. No obstante, no es lo mismo empleados que cooperan (participan), dado los incentivos correctos de remuneración y reconocimiento, a asociados que trabajan de manera cooperativa (colaboran). Jerárquicamente la meta de una empresa privada es la rentabilidad y lo demás, son medios para obtener su objetivo principal. En cambio, la meta principal de una entidad de economía solidaria es el bienestar social, lo demás, incluido la utilidad, son medios para conseguir su objetivo principal. Goldratt et al. (2008) expone que en los negocios es muy fácil determinar la meta con dinero, pero, en las organizaciones que no persiguen fines de lucro, la meta se debe medir en términos no monetarios (p. 223). Cambiar los fines, establece una manera distinta de pensar la estructura de gobierno de la fabricación, administración y comercialización de las entidades económicas.

Con este factor superaban las limitaciones y carencias de todos y cada uno de los demás factores, inyectándoles un plus de energía social y de eficiencia que explicaba gran parte del producto. Las carencias tecnológicas las superaban compartiendo conocimientos fragmentarios y trabajando en equipo en la búsqueda de soluciones a los problemas técnicos: es impresionante la creatividad personal y colectiva que se genera cuando las 
personas se unen para enfrentar una dificultad que deben resolver en común. (Valencia y Gallego, 2013, p. 71)

El capital humano es compensado con remuneración. Todos los empleados cooperan para cumplir el objetivo de los dueños, por un salario. La inversión en el capital humano se puede realizar con capacitación de los empleados, mayor remuneración, comisiones, regalos, etc. Pero esto no necesariamente se ve representado en solidaridad (Razeto, 2007b). La empresa privada, para garantizar su inversión, desarrolla contratos de aseguramiento, para recuperar los egresos aportados a los empleados e invierte en publicidad para hacer visible su altruismo. Nada es realizado por fuera del principal objetivo: rentabilidad.

En las empresas verdaderamente solidarias, existen otro tipo de relaciones de trabajo, algunos no reciben compensación monetaria, pues se trabaja para la apropiación del derecho comunitario y el beneficio mutuo. Son dueños de sus organizaciones, de libre adhesión, que deciden la administración de su entidad de manera democrática, basados en sus principios éticos solidarios (Vélez-Tamayo, 2014). "El sector cooperativo necesita de un mayor reconocimiento al exterior por parte de los mercados de las bondades de su modelo organizacional como ventaja competitiva" (Ruiz y Quesada, 2014, p. 897).

Una empresa privada puede invertir en el bienestar de los empleados, pero será un medio para cumplir con el objetivo financiero, pues en el momento de una crisis económica, los empleados serán los menos beneficiados en las decisiones. En cambio, en una empresa verdadera de economía solidaria, en tiempos de dificultad económica, lo principal no será la rentabilidad, sino el bienestar común (Sabín et al., 2013). El factor C crea capital solidario, que servirá para potencializar el capital humano, estructural, relacional y financiero.

\section{Discusión}

Los trabajos sobre la caracterización del CI confluyen en tres factores: capital humano, estructural y relacional. La literatura de los factores presentados para la caracterización del CI, usualmente no toma en cuenta el contexto de las organizaciones solidarias, donde los factores planteados no 
alcanzan a definir y explicar los elementos intangibles motivadores de los resultados de estas organizaciones (Escobar et al., 2018; Ruiz y Quesada, 2014).

Este artículo de revisión de la literatura exhibe que, para las empresas de economía solidaria, hay un elemento adicional de los intangibles formados, que añade valor y que no se encuentra caracterizado en los factores relacionados en la literatura de CI. Por lo tanto, se deja a consideración la caracterización del CI específicamente para las empresas de economía solidaria en los siguientes factores: capital humano, estructural, relación y solidario (este último explicado por la literatura del factor C).

Esta revisión halló un cuerpo de literatura que examina el factor C, cuya idea agrupa la existencia de elementos intangibles en relación con los asociados, que se derivan directamente de la unión solidaria, y que es determinante en los resultados de las entidades cooperativas (Agudelo et al., 2010; Colls y Bravo, 2012; Dávila et al., 2018; Gallego, 2015; Jiménez, 2016; Pérez y Etxezarreta, 2015; Pulido-Garzón et al., 2019; Sabín et al., 2013; Valencia y Gallego, 2013; Vélez-Tamayo, 2014).

Es importante que las organizaciones de economía solidaria, separen y conceptualicen el capital solidario, para gestionarlo de manera independiente de los otros factores económicos. La calidad de este factor está directamente relacionada con el cumplimiento del objetivo de bienestar común. Este trabajo encontró una relación estrecha entre lo que Razeto (2015) llama factor C y el capital solidario. La literatura plantea que entre más calidad contenga el factor C, mayor será el crecimiento esperado del bienestar común en las empresas de economía solidaria, y viceversa.

\section{Conclusiones}

Las organizaciones de economía solidaria promueven el bienestar común de sus miembros y colaboradores, es ahí donde radica esencialmente su fortaleza. En la economía utilitarista, el fin principal es la rentabilidad financiera, en cambio, en la economía solidaria, la rentabilidad es un medio. La creación de valor de las organizaciones está relacionada con el cumplimiento de sus fines principales, los cuales son representados en activos tangibles e intangibles, siendo este último

el más complejo de caracterizar. El campo de estudio de los activos intangibles es conocido como 
capital intelectual, en el que se clasifican: el capital humano, estructural y relacional; sin embargo, los factores expuestos no consiguen explicar los resultados de las organizaciones solidarias, por tanto, se plantea la existencia de otro factor. A partir de ello se desarrolló un marco conceptual que propone caracterizar el concepto de capital solidario dentro de la estructura del capital intelectual en las empresas de economía solidaria.

Este documento contribuye a la literatura sobre las categorías del CI, separando y clasificando el capital solidario como un intangible importante en las empresas de economía solidaria. No obstante, este trabajo no ofrece estrategias ni métodos de valoración del capital solidario, cuestión que no está dentro del alcance de este artículo, la continuidad en estos temas exige un trabajo colaborativo de la academia y de las entidades de economía solidaria.

\section{Referencias}

(1) Agudelo, M. F., Patiño, B. L., \& Ramos Bravo, M. A. (2010). Conceptualización del factor C. Antecedentes y perspectivas. Ingeniería Solidaria, 6(10-11), 95-01. https://revistas.ucc.edu.co/index.php/in/article/view/456

(2) Andreou, A. N., Green, A., \& Stankosky, M. (2007). A framework of intangible valuation areas and antecedents. Journal of Intellectual Capital, 8(1), 52-75. https://doi.org/10.1108/14691930710715060

(3) Andriessen, D. (2001). Weightless wealth: Four modifications to standard IC theory. Journal of Intellectual Capital, 2(3), 204-214. https://doi.org/10.1108/14691930110399941

(4) Aparicio, P. G. (2003). El capital social en las sociedades cooperativas . Las normas sobre los aspectos contables de las sociedades cooperativas a la luz de los principios cooperativos. CIRIEC-España, Revista de Economia Pública, Social y Cooperativa, (45), 57-79. https://core.ac.uk/download/pdf/33107372.pdf

(5) Arriagada, I. (2006). Breve guía para la aplicación del enfoque de capital social en los programas de pobreza. Comisión Económica para América Latina y el Caribe. https://agris.fao.org/agris-search/search.do?recordID=XF2015038681 
(6) Bueno, E. (2002). El capital social en el nuevo enfoque del capital intelectual de las organizaciones. Revista de Psicología del Trabajo y de las Organizaciones, 18(2-3), 157-176. http://www.redalyc.org/articulo.oa?id=231318274003

(7) Campos, A. (2008). Una aproximación al concepto de "lo social" desde trabajo social. Revista Tendencias \& Retos, (13), 55-70. http://www.ts.ucr.ac.cr/binarios/revistas/co/rev-cotendencias-0013-05.pdf

(8) Castells, M. (2002). La era de la información: economía, sociedad y cultura (4ª ed). Siglo XXI Editores Argentina S.A.

(9) Chen, M-C., Cheng, S-J., \& Hwang, Y. (2005). An empirical investigation of the relationship between intellectual capital and firms' market value and financial performance. Journal of Intellectual Capital, 6(2), 159-176. https://doi.org/10.1108/14691930510592771

(10)Colls, Y., \& Bravo, M. T. (2012). Los factores productivos desde la prisma del factor C. Revista Momboy, 60-83.

(11) Currás, E. (2005). Ontologías, taxonomía y tesauros. Manual de construcción y uso (3 $3^{\mathrm{a}}$ ed). Gijón.

(12) Dávila, R., Vargas, A., Blanco, L., Roa, E., Cáceres, L. E., \& Vargas, L. A. (2018). Characteristics of the Colombian solidarity economy. Approaches to influential currents in Colombia. CIRIEC-Espana Revista de Economia Publica, Social y Cooperativa, (93), 85113. https://doi.org/10.7203/CIRIEC-E.93.10327

(13) Decreto 2650 de 1993. (1993, 29 de diciembre). Presidencia de la República de Colombia. Diario oficial No. 41156. http://www.suin-juriscol.gov.co/viewDocument.asp?id=1772403

(14)Delgadillo, D. I. (2001). El sistema de información contable. Universidad del Valle.

(15)Edvinsson, L. (1997). Developing intellectual capital at Skandia. Long Range Planning, 30(3), 366-373. https://doi.org/10.1016/S0024-6301(97)90248-X

(16) Escobar, A., Velandia, G., \& Navarro, E. (2018). Gestión del conocimiento y competitividad en las cooperativas con sección de ahorro y crédito. REVESCO. Revista de Estudios Cooperativos, 127, 90-115. https://doi.org/10.5209/reve.59769

(17)Funes, Y., \& Hernández, C. (2001). Medición del valor del capital intelectual. Contaduría y Administración, (203), 45-58. https://www.redalyc.org/pdf/395/39520305.pdf

(18) Gallego, L. A. (2015). Análisis desde la Teoría Económica Comprensiva: el Factor Gestión y el Factor Comunidad, en las organizaciones de pequeños y medianos productores 
agropecuarios. El caso de la subregión del Altiplano en el Oriente Antioqueño. [Tesis de maestría, Universidad Nacional de Colombia]. https://repositorio.unal.edu.co/handle/unal/54880?show=full

(19) Galleguillos-Cortés, C., Silva-Muna, J. L., \& Becerra-Muñoz, P. (2018). Incidencia del Capital Intelectual en el rendimiento financiero de las Universidades de Chile. Ingeniare. Revista Chilena de Ingeniería, 26(4), 593-598. https://doi.org/10.4067/s071833052018000400593

(20) Goldratt, E. M. (1994). El síndrome del pajar. Ediciones Castillo.

(21) Goldratt, E. M., Schragenheim, E, \& Ptak, C. A. (2008). Necesario pero no suficiente. Granica.

(22) González, P. (2017). Una propuesta para medir la creación de valor por parte del capital intelectual en grandes empresas colombianas. Harvard Deusto Business Research, 6(1), 3-16. https://doi.org/10.3926/hdbr.135

(23) Iazzolino, G., \& Laise, D. (2013). Value added intellectual coefficient (VAIC): A methodological and critical review. Journal of Intellectual Capital, 14(4), 547-563. https://doi.org/10.1108/JIC-12-2012-0107

(24) Iazzolino, G., \& Laise, D. (2016). Value creation and sustainability in knowledge-based strategies. Journal of Intellectual Capital, 17(3), 457-470. https://doi.org/10.1108/JC-092015-0082

(25)IFRS Foundation. (2019). NIC 38 Activos intangibles. UK IFRS Foundation. https://acortar.link/1bk5

(26) Ijiri, Y. (1967). The foundation of accounting measurement. Scholars Book Co.

(27) Jiménez, J. (2016). Movimiento de Economía Social y Solidaria de Ecuador. Circuitos Económicos Solidarios Interculturales. Revista de La Academia, (21), 101-128. https://doi.org/10.25074/0196318.0.58

(28) Keong Choong, K. (2008). Intellectual capital: definitions, categorization and reporting models. Journal of Intellectual Capital, 9(4), 609-638. https://doi.org/10.1108/14691930810913186

(29) Mačerinskienè, I., \& Survilaite, S. (2019). Company's intellectual capital impact on market value of Baltic countries listed enterprises. Oeconomia Copernicana, 10(2), 309-339. 
https://doi.org/10.24136/oc.2019.016

(30) Marcuello Servos, C., \& Saz Gil, M. I. (2008). Los principios cooperativos facilitadores de la innovación: un modelo teórico. REVESCO. Revista de Estudios Cooperativos, 94, 59-79.

(31) Mattessich, R. (2002). Contabilidad y métodos analíticos. Medición y proyección del ingreso y la riqueza en la microeconomía y en la macroeconomía. Fondo editorial La Ley.

(32) Millán, R., \& Gordon, S. (2004). Capital social: una lectura de tres perspectivas clásicas. $\begin{array}{llll}\text { Revista Mexicana } \quad \text { de } & \text { 711-747. }\end{array}$ http://www.scielo.org.mx/pdf/rms/v66n4/v66n4a4.pdf

(33) Nazari, J. A., \& Herremans, I. M. (2007). Extended VAIC model: measuring intellectual capital components. Journal of Intellectual Capital, 8(4), 595-609. https://doi.org/10.1108/14691930710830774

(34) Ostrom, E., \& Ahn, T. K. (2003). Una perspectiva del capital social desde las ciencias sociales: capital social y acción colectiva. Revista Mexicana de Sociología, 65(1), 155-233. http://www.scielo.org.mx/pdf/rms/v65n1/v65n1a5.pdf

(35)Packer, M. (2013). La ciencia de la investigación cualitativa. Ediciones UniandesUniversidad de los Andes.

(36) Pedro, E., Leitão, J., \& Alves, H. (2018). Intellectual capital and performance: Taxonomy of components and multi-dimensional analysis axes. Journal of Intellectual Capital, 19(2), 407452. https://doi.org/10.1108/JIC-11-2016-0118

(37) Pérez, J. C., \& Etxezarreta, E. (2015). Otros modelos de empresa en la economía solidaria: entre la retórica y la práctica. Lan Harremanak, (33), 227-252. https://doi.org/10.1387/lanharremanak.16110

(38) Pulic, A. (2008). The principles of intellectual capital efficiency - A brief description. Croatian Intellectual Capital Center, 76, 1-24. https://acortar.link/1bop

(39) Pulido-Garzón, A., Guerrero-Julio, M. L., \& Celis-Patiño, Y. (2019). Impacto de las relaciones humanas en la competitividad de las asociaciones. Revista UIS Ingenierías, 18(1), 61-72. https://doi.org/10.18273/revuin.v18n1-2019005

(40) Ragab, M., \& Arisha, A. (2013). Knowledge management and measurement: a critical review. Journal of Knowledge Management, 17(6), 873-901. https://doi.org/10.1108/JKM-12-20120381

(41) Razeto Migliaro, L. (1996). Los caminos de la economía de solidaridad. Lumen, Humanitas. 
http://lacoperacha.org.mx/documentos/coperacha-economia-solidaria-razeto.pdf

(42)Razeto, L. (2007). Factor C. Retrieved December 12, 2019, from http://www.economiasolidaria.org/files/el_factor_c.pdf

(43) Razeto, L. (2007b). La economía de solidaridad: concepto, realidad y proyecto. En: J. L. Coraggio. (Ed.), La economía social desde la periferia. Contribuciones latinoameicanas (pp. 317-338). Editorial Altamira. https://cutt.ly/vyQmyr8

(44)Razeto, L. (2015). Tópicos de economía comprensiva. Ediciones Univérsitas Nueva Civilización. https://www.luisrazeto.net/content/t\%C3\%B3picos-de-econom\%25C3\%25ADcomprensiva

(45) Reilly, R., \& Schweihs, R. (1998). Valuing intangible assets. McGraw Hill.

(46) Rodov, I., \& Leliaert, P. (2002). FiMIAM: financial method of intangible assets measurement. Journal of Intellectual Capital, 3(3), 323-336. https://doi.org/10.1108/14691930210435642

(47) Ruiz, I., \& Quesada, J. M. (2014). Los principios cooperativos como capital intangible ante los desafíos del cooperativismo. Intangible Capital, 10(5), 897-921. https://doi.org/10.3926/ic.406

(48) Sabín, F., Fernández, J., \& Bandrés, I. (2013). Factor C: Factores de resistencia de las microempresas cooperativas frente a la crisis y recomendaciones para un fortalecimiento cooperativo del sector de lo social. Revista Vasca de Economía Social, (9), 74-100. https://dialnet.unirioja.es/servlet/articulo?codigo $=4521525$

(49) Smith, A. (1776). La riqueza de las naciones. Traducción Carlos Rodríguez Braun (1996). ePubLibre.

(50) Ståhle, P., Ståhle, S., \& Aho, S. (2011). Value added intellectual coefficient (VAIC): a critical analysis. Journal of Intellectual Capital, 12(4), 531-551. https://doi.org/10.1108/14691931111181715

(51) Survilaitè, S., Tamošiūnienè, R., \& Shatrevich, V. (2015). Intellectual Capital Approach to Modern Management through the Perspective of a Company's Value Added. Verslas: Teorija Ir Praktika, 16(1), 31-44. https://doi.org/10.3846/btp.2015.553

(52) Tamošiūnienè, R., Survilaitè, S., \& Shatrevich, V. (2015). Intellectual capital approach to modern management through the perspective of a company's value added. Business: Theory \& Practice, 16(1), 31-44. https://doi.org/10.3846/btp.2015.553 
(53) Valencia, L. M., \& Gallego, L. A. (2013). Factor C-comunidad, factor dinamizador en las organizaciones solidarias. Cooperativismo \& Desarrollo, 21(103), 69-80. https://doi.org/10.16925/co.v21i103.676

(54) Vélez-Tamayo, J. M. (2014). Construcción del concepto de economía solidaria: una mirada a la teoría económica comprensiva. Cooperativismo \& Desarrollo, 22(105), 17-29. https://doi.org/10.16925/co.v22i105.690

(55) Vera Carrasco, O. (2009). Cómo Escribir Artículos De Revisión. Rev Med La Paz, 15(1), 6369.

(56) Walde, V. R. (2011). El capital social en las diversas formas de la sociedad anónima, los principios y las diversas funciones que cumple tanto externa como internamente en estas. Revista Oficial del Poder Judicial, 4-5(6-7), 47-68. https://acortar.link/1bk6

Cómo citar este artículo: Rincón, C., Molina, F. y González, P. (2021). El capital intelectual en las organizaciones de economía solidaria. Tendencias, 22(2), 309-330. https://doi.org/10.22267/rtend.212202.178 